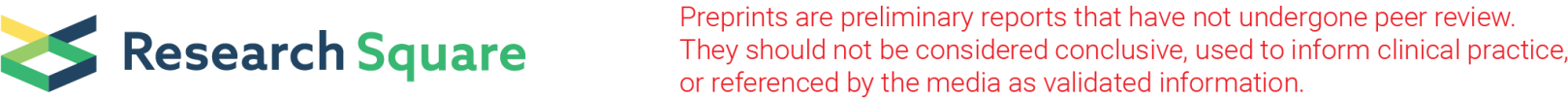

\section{Caring for older dependent people in low and middle-income countries: the effect of age on mental health in caregivers}

Ruttana Phetsitong ( $\nabla$ ruttana.phe@mahidol.ac.th )

Mahidol University https://orcid.org/0000-0003-2442-4804

Patama Vapattawong

Mahidol University

Rosie Mayston

King's College London

Martin Prince

King's College London

Kia-Chong Chua

King's College London

Research article

Keywords: Ageing, Caregiver, Mental health, Burden, Developing countries

Posted Date: July 21st, 2020

DOl: https://doi.org/10.21203/rs.3.rs-40311/v1

License: (c) (1) This work is licensed under a Creative Commons Attribution 4.0 International License.

Read Full License 


\section{Abstract}

Background: Older people worldwide are living with chronic health problems, of whom $80 \%$ were from low and middle-income countries. Caregiver stress is a complex interplay of health and socio-economic factors with older caregivers, however, the effects of caregiver age upon their mental health have not been well-described. This study aimed to examine whether caregiver age predicts their mental health outcomes in terms of psychological morbidity and care burden in Latin America and China.

Methods: The present study was based from cross-sectional baseline data of seven low and middleincome countries including; Cuba, Dominican Republic, Peru, Venezuela, Mexico, China, and Puerto Rico, collected between February 2003 and June 2009 by the 10/66 Dementia Research Group. Data from 1348 households where the caregiver provided care for one older person in the household were included in the current analysis. Psychological morbidity was assessed through the Self-Reporting Questionnaire. The Zarit Burden Inventory was used to measure the levels of care burden of caregivers. Multivariate logistic regression was used to investigate the effects of caregiver age upon psychological morbidity and care burden. Meta-analysis was performed to obtain pooled estimating one overall odd ratios and $95 \%$ confidence intervals of the analyses from the different countries, using the inverse-variance weighted fixed-effect method.

Results: The prevalence of the household with older caregivers is almost the same level as of the households with younger caregivers in Puerto Rico and China. The models unadjusted and adjusted for all potential covariates (household size, number of household asset, caregiver sex, caregiver marital status, caregiver education, cognitive impairment of older person, functional dependency of older person, behavioral problem of older person, and caregiving hours) showed that there was no statistically significant difference in psychological morbidity and care burden between older caregivers and non-older caregivers across countries. The Adjusted pooled estimates, however, indicated lower psychological morbidity among older caregivers (OR $\left.=0.61,95 \% \mathrm{Cl}: 0.41-0.93, \mathrm{I}^{2}=0.0 \%\right)$.

Conclusions: Older caregivers are at lower risk of mental health problem than younger caregivers. This information may help to provide a greater understanding for service support, policy makers and health care providers.

\section{Background}

The projected increase in the burden of chronic diseases in low and middle-income countries (LMICs) is largely driven by the underlying determinants of rapid population ageing. It has been estimated that 70 million older people worldwide are living with chronic health problems, of whom $80 \%$ were from LMICs [1]. Moreover, the prevalence of chronic disease is rising quickly across LMICs [2].

The proportion of the population aged 65 years or older in LMICs is projected to double between 2019 and 2050 [3]. Along with the population aging situation, one of the major concerns is that both care receivers and caregivers are growing older. Current demographic trends such as decreases in household 
sizes, higher occurrences of divorce, and greater geographic distances between families, have limited the availability of caregivers [4]. As a result, spouses and older adult children of the older people are progressively becoming responsible for caregiving in LMICs $[5,6]$.

Caregiving stress and burden are inevitable parts of being a caregiver, which adversely prevail their physical and mental health, financial as well as being a social burden $[7,8]$. Caregivers' mental health problems have been recognised as a crucial health risk and mortality determinants for both caregivers and care receivers [9]. Caregiver burden is a complex interaction of caregivers' demographic and socioeconomic factors and the health conditions of the people being cared of.

Compared with younger caregivers, older caregivers tend to have a double-burden due to their age which is related to physical and psychological morbidity and lower employment [10-12]. The older caregivers who are financially supporting other household members, and those with economically inactive or financial strain reported psychological problems $[13,14]$. However, it was evident that better preparedness (perceived readiness) for caregiving role interacting with high mutuality (relationship quality), which was normally found in older spouse caregivers, buffer caregivers' psychological distress responses to high caregiver demands [15]. The closer relationships provided positive outcomes for the caregivers such as compromise, problem-solving skills, and positive adjustment [16]. In addition, the impact of caregiver age upon caregiver mental burden might be different across cultures, particularly in LIMICs society due to social expectations and long-term care availability [17].

Existing studies from the 10/66 Dementia Research Group (DRG) population-based surveys of dementia and ageing in low and middle-income countries provides evidence to suggest that caring for older dependent people is associated with substantial mental health problems in caregivers [18-20]. To the author's knowledge, the effects of caregiver age upon their mental health have not been well-described. To address this gap in the current literature on caregiving in the context of the 10/66 DRG surveys and our understanding of these challenges, the objective of this study is to examine how caregiver's age group at baseline predicts their mental health in Latin America and China.

As mentioned previously, demographic change has contributed to a decrease in available young caregivers resulting in older caregivers having to serve this function. It presents a double-burden for older caregivers due to their age which related stress and morbidity. This study, therefore, aimed to test two hypotheses; (i) older caregivers experience worse psychological morbidity compared to younger caregivers, and (ii) older caregivers experience worse care burden compared to younger caregivers.

\section{Methods}

\section{Study design, settings and sample}

The 10/66 DRG has carried out a series of repeated cross-sectional surveys in Cuba, Dominican Republic, Peru, Venezuela, Brazil, Mexico, Puerto Rico, China, and India with the aim of understanding the health of older people with dementia in low and middle-income countries. This was conducted in older residents 
aged 65 years and over and their caregivers, living in eleven geographically defined urban and rural catchment area sites. Details of study methodology and protocols for the 10/66 DRG have been described elsewhere [21]. A full assessment was conducted for all eligible older individuals if they, or their kin, agreed to participate in the survey. The assessment covers a background socio-demographic, health and risk factor interview, a structured clinical mental state assessment, and physical examination.

The present study was based from the $10 / 66$ baseline data from catchment area sites in seven low and middle-income countries including; Cuba, Dominican Republic, Peru, Venezuela, Mexico, China, and Puerto Rico, conducted between February 2003 and June 2009 (in 2003-2006 for Cuba; in 2003-2004 for Dominican Republic; in 2005-2007 for Peru; in 2004-2006 for Venezuela; in 2005-2007 for Mexico; in 2003-2005 for China; and in 2006-2009 for Puerto Rico). The study sites consisted of urban and rural areas. The boundaries of each catchment area were precisely defined, and households were assigned an identification number. Each household was then systematically visited to identify all household members aged 65 years and over who were eligible to participate in the survey. The specific details of the data of each country were have been published elsewhere [22]. The total sample of households with older people from the seven countries was 11,717 .

\section{Selected households and participants}

This study defined a main caregiver as a person who provides most 'hands on' care or the family member who is the main 'organisational' caregiver. Data from 1348 households, where the main caregiver provided care for one older person diagnosed with dementia or without dementia in the household, were included in the current analysis. In order to test the research hypotheses, the sample households were classified into two categories; (i) younger caregiver households (where the main caregiver of the household was aged 64 years or lower), and (ii) older caregiver households (where the main caregiver of the household was aged 65 years or over).

\section{Measures of caregiver mental health}

Psychological morbidity was assessed through Self-Reporting Questionnaire (SRQ). The questionnaire has been widely used in different populations. There are 20 items covering symptoms of depression, anxiety, and somatised distress [23]. The SRQ detects the presence of non-psychotic psychological symptoms over the past two weeks. It yields a total score that ranges from 0 to 20 . Recommended by the WHO [24], higher scores indicating higher psychological morbidity with a cut-off score $\geq 8$ is identified as psychological morbidity (coded as 1 ), whereas scores of less than 8 were coded as 0 for nonpsychological morbidity.

Care burden was measured using the Zarit Burden Inventory (ZBI) which is widely cited in caregiver burden literature and has been translated and validated allowing for international comparisons. It was used in the 10/66 pilot studies of the listed countries of this study, strong psychometric properties and cultural relevance were found [18]. The ZBI consists of 22 items including; health, psychological wellbeing, social life, finances, and the relationship between the caregiver and the older person, with each 
item being evaluated using a 5-point Likert scale [25]. The cut-off scores from 41 to 88 indicates "a high burden" (coded as 1), and the scores below 41 signify "a low burden" (coded as 0 ) [25, 26].

\section{Measures of covariate variables}

Guided by the Pearlin Stress Process Model [27] and the Yates Stress Process Model [28]. The study sought to control for potential factors including household characteristics, older people's health status, and socio-demographic status of the caregivers and caregiving hours. These were entered into the model analyses as potential confounding factors, since these variables might affect caregiver's psychological distress and care burden.

\section{Household characteristics}

Household size refers to number of household members of the older people household. It is often associated with considerable mental health due to long term care for older people in the household [29].

Number of assets in household out of a possible total list of seven (television, fridge/freezer, mains water, mains electricity, telephone, plumbed toilet, plumbed bathroom) is a proxy of socioeconomic status of the household since it represents more permanent status than does either incomes or consumption [30].

\section{Older people's health status}

Dementia refers to the presence of dementia according to the 10/66 Dementia Diagnostic assessments (the CERAD 10-word list recall task, the Community Screening Instrument for Dementia; CSI-D, and the Geriatric Mental State examination; GMS) or DSM-IV dementia criteria. Presenting dementia either measured by the three 10/66 assessments or the DSM-IV was coded as 1, and 0 for non-presence dementia assessed by both measurements.

Functional dependency is the severe difficulty performing basic Activities of Daily Living; ADLs (eating, dressing, toileting, bathing, and walking) assessed by the WHO-DAS developed by WHO. This measure has shown promise in predicting disability level of older people affecting care burden [31]. The severe difficulty with three or more basic $A D L$ was coded as 1 , and 0 for the severe problem with lower than three.

Behavioural problems is the severity of 12 behavioral problems including presence of delusions, hallucinations, agitation/aggression, depression, anxiety, irrational euphoria, apathy, disinhibition, irritability, motor disturbance, disruptive nighttime behavior, and adverse appetite/eating change. The measurement is based on the Neuropsychiatric Inventory-Questionnaire (12NPI-Q) score, administered to the main caregiver.

\section{Socio-demographic characteristics of the caregivers and caregiving hours}

Sex refers to sex of the main caregiver. Sex difference is mentioned frequently in the literature, it is related to kinship role and perception of stress and caregiver burden [32]. 
Marital status refers to the marital status of the main caregiver at the time of the survey. Theory and empirical findings suggest that there is a relationship between marital status and stress or mental health $[33,34]$. It was coded for this analysis as a partnership (currently married or cohabiting) versus nonpartnership (single /widowed/divorced/separated).

Education level is the highest level of completed education of the main caregiver. Caregivers with a relatively low education could be assumed to report a higher burden [35]. In this study, it was categorized into primary or lower and higher than primary.

Caregiving hours is the time spent on assisting the older person with ADLs (eating, dressing, toileting, bathing, communicating, using transportation, looking after one's appearance, and supervising) by the main caregiver in the last 24 hours. The more confining the caregiver task, the more likely that it will create adverse mental health consequences for the caregiver [36, 37].

\section{Statistical Analyses}

Descriptive analysis was employed to define the sample, to analyse the distribution of the background variables, and to explore the quantitative level of variables. Tests of significance were performed to examine the association between the household and caregiver background variables, and the health status variables of the older persons with caregiver age group using chi-square tests or Mann-Whitney $U$ tests.

The main strategy for analysis was to apply multivariate models based on binary logistic regression to investigate the effects of caregiver age upon psychological morbidity and care burden. The first model was unadjusted model. All covariate variables (household size, number of household asset, caregiver sex, caregiver marital status, caregiver education, cognitive impairment of older person, functional dependency of older person, behavioral problem of older person, caregiving hour) were controlled in the second model. An odd ratio (OR) and $95 \%$ confidence interval (Cl) were presented to determine whether a caregiver age is a risk factor for psychological morbidity and care burden, and to compare the magnitude of the effect of caregiver age on the psychological morbidity and care burden. Meta-analysis was performed to obtain pooled estimating one overall OR and $95 \% \mathrm{Cl}$ of the analyses from the different countries, using the inverse-variance weighted (fixed-effect) method. All statistical analyses were performed using STATA/SE 14.0 (StataCorp, Texas, USA).

\section{Results}

\section{Sample characteristics}

In total, one thousand three hundred and forty-eight older people households were included in the study. The prevalence of older caregiver households, where an older person in the household received care from a main older caregiver, was broadly similar across the seven countries (Table 1). The households with older caregivers were less prevalent compared to younger caregiver households. Notably, in China and 
Puerto Rico, $40.2 \%$ and $45.2 \%$ respectively, were older caregiver households. The characteristics of the households, background variables for the older people, and socio-demographic of caregivers and caregiving hours between the caregiver age group by country are summarized in Tables 1 and 2 .

The findings showed neither household characteristics nor health status in older people was significantly different between younger and older caregivers. In addition, there was no significant median difference in caregiving hours between older and younger caregivers. In regard to caregiver characteristics, the majority of the caregivers were female across the different countries. The association of sex and caregiver age was statistically significant in Peru, Mexico, and Puerto Rico. According to marital status, a higher prevalence of partnership caregivers was shown in both younger and older caregiver households, in China in particular. In contrast, the majority of both younger and older caregivers in Puerto Rico were nonpartnership. In terms of caregiver's education, the bivariate analysis indicated that there were significant differences between younger and older caregivers across the seven countries, with younger caregivers having a higher education status than older caregivers. In Dominican Republic, Venezuela, Mexico, and China, there was a higher prevalence of older caregivers being educated at primary school or less, whereas the majority of older caregivers in Cuba, Peru, and Puerto Rico had an educational level higher than primary level. 
Table 1

Prevalence of older caregiver households vs. younger caregiver household, and background characteristics of households, health status of the older people between the caregiver age by country $(\mathrm{n}=$ 1348 households)

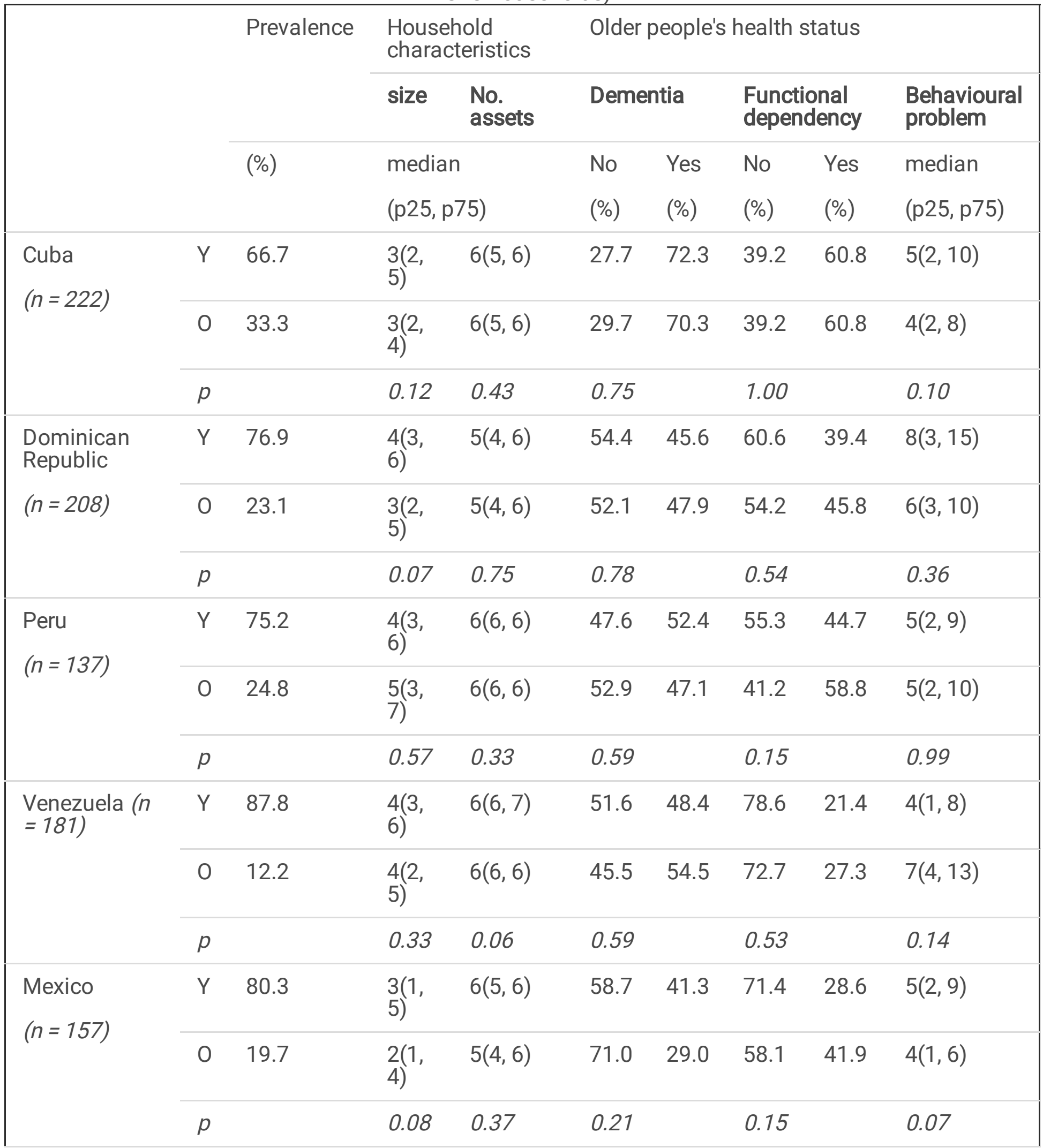

Note: $Y=$ younger caregiver; $\mathrm{O}=$ older caregiver; $p=p$-value; $\mathrm{p} 25=$ the 25 th percentile; $\mathrm{p} 75=$ the 75 th percentile 


\begin{tabular}{|c|c|c|c|c|c|c|c|c|c|}
\hline \multirow{3}{*}{$\begin{array}{l}\text { China } \\
(n=204)\end{array}$} & Y & 59.8 & $\begin{array}{l}3(2, \\
4)\end{array}$ & $6(5,7)$ & 55.7 & 44.3 & 54.1 & 45.9 & $0(0,2)$ \\
\hline & 0 & 40.2 & $\begin{array}{l}3(2, \\
5)\end{array}$ & $5(5,6)$ & 57.3 & 42.7 & 48.8 & 51.2 & $0(0,1)$ \\
\hline & $p$ & & 0.69 & 0.28 & 0.82 & & 0.46 & & 0.72 \\
\hline \multirow[t]{3}{*}{$\begin{array}{l}\text { Puerto Rico } \\
(n=239)\end{array}$} & $\mathrm{Y}$ & 54.8 & $\begin{array}{l}2(2, \\
3)\end{array}$ & $7(6,7)$ & 44.6 & 55.4 & 44.3 & 55.7 & $3(0,8)$ \\
\hline & 0 & 45.2 & $\begin{array}{l}2(2, \\
3)\end{array}$ & $7(6,7)$ & 42.1 & 57.9 & 47.2 & 52.8 & $3(0,6)$ \\
\hline & $p$ & & 0.43 & 0.46 & 0.69 & & 0.65 & & 0.23 \\
\hline
\end{tabular}


Table 2

Socio-demographic background of caregivers and caregiving hours between the caregiver age by country ( $n=1348$ households)

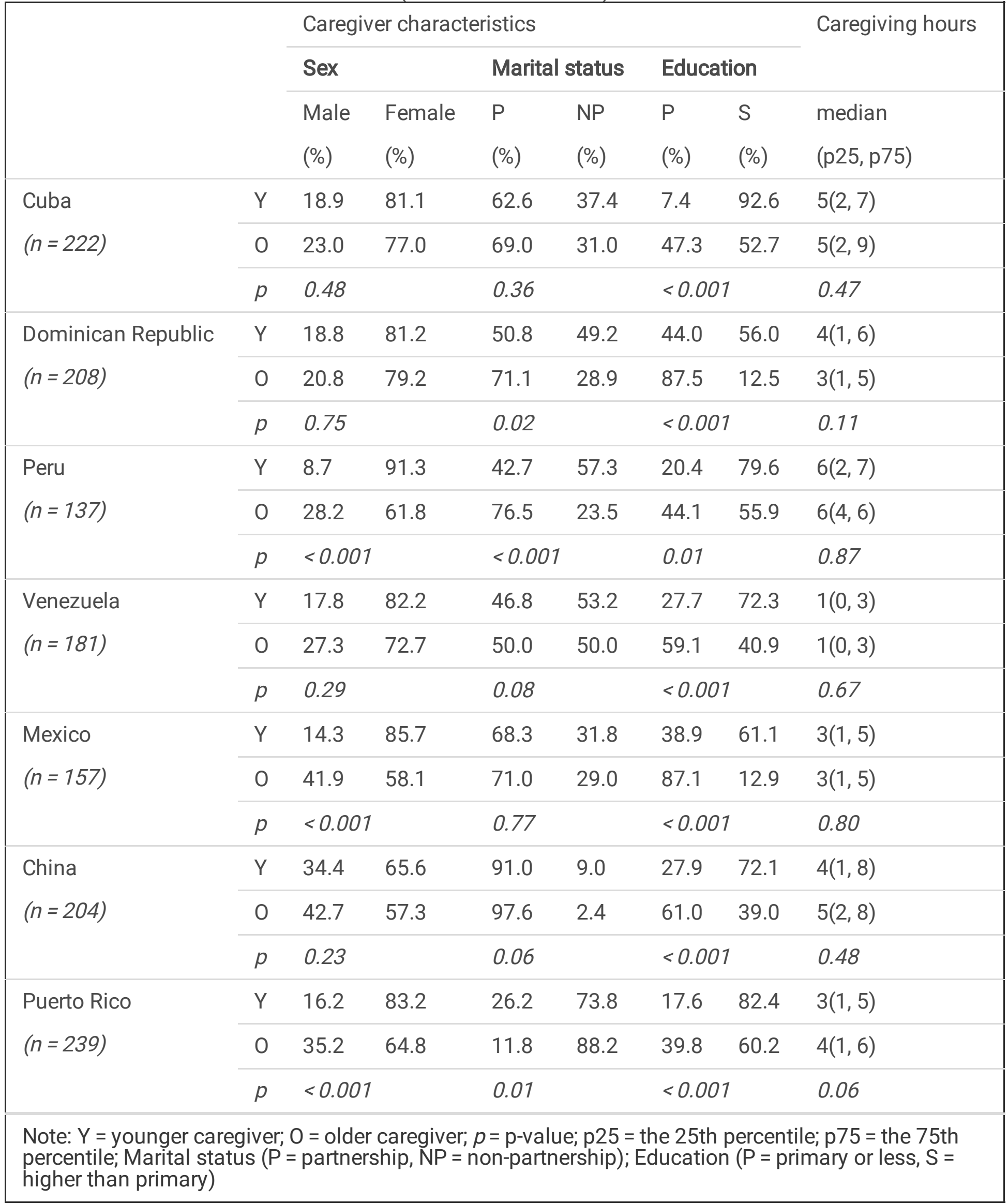




\section{The effects of caregiver age upon psychological morbidity and care burden}

To investigate the effect of caregiver age group (older caregivers vs. non-older caregivers) upon their psychological morbidity and care burden alongside other potential confounders, therefore a multivariate analysis based on binary logistic regression was used, Table 3 .

In model I, the unadjusted analysis showed no significant differences in caregivers' mental health outcomes in terms of psychological morbidity and care burden between younger and older caregivers across the countries, and the pooled estimates remained unaffected. The adjusted analysis (model II), also showed no statistically significant differences between younger and older caregivers in all countries. However, the pooled estimates showed that psychological morbidity were significantly associated with caregiver age $\left(\mathrm{OR}=0.61,95 \% \mathrm{Cl} 0.41-0.93, \mathrm{I}^{2}=0.0 \%\right)$, with older caregivers being at $39 \%$ less risk of psychological morbidity than younger caregivers. It is worth noting that the odds ratio of the psychological morbidity in China was larger compared to Latin America, although this was not significant. 
Table 3

Unadjusted and adjusted association between older caregiver and caregiver psychological morbidity and care burden

\begin{tabular}{lllllll} 
Country & \multicolumn{4}{l}{ Psychological morbidity } & \multicolumn{3}{c}{ Care burden } \\
\cline { 2 - 7 } & OR & $95 \% \mathrm{Cl}$ & OR & OR & $95 \% \mathrm{Cl}$
\end{tabular}

lower upper lower upper

Model I (unadjusted model)

\begin{tabular}{|lcccccccc|}
\hline Cuba & 222 & 0.60 & 0.30 & 1.20 & 220 & 1.12 & 0.57 & 2.23 \\
\hline Dominican Republic & 208 & 0.85 & 0.41 & 1.78 & 206 & 0.80 & 0.28 & 2.27 \\
\hline Peru & 137 & 0.78 & 0.36 & 1.69 & 137 & 0.94 & 0.32 & 2.78 \\
\hline Venezuela & 181 & 1.50 & 0.51 & 4.44 & 178 & 1.43 & 0.44 & 4.63 \\
\hline Mexico & 157 & 0.52 & 0.18 & 1.46 & 156 & 0.43 & 0.05 & 3.52 \\
\hline China & 204 & 6.21 & 0.68 & 56.55 & 204 & 0.67 & 0.27 & 1.63 \\
\hline Puerto Rico & 239 & 0.65 & 0.33 & 1.30 & 239 & 0.38 & 0.12 & 1.22 \\
\hline Pooled estimate & & 0.77 & 0.56 & 1.06 & & 0.84 & 0.58 & 1.24 \\
\hline Heterogeneity P & & $3.7 \%$ & & & & $0.0 \%$ & & \\
\hline Model II (adjusted model) & & & & & & & \\
\hline Cuba & 208 & 0.48 & 0.20 & 1.14 & 206 & 1.17 & 0.50 & 2.76 \\
\hline Dominican Republic & 175 & 0.82 & 0.33 & 2.05 & 173 & 1.30 & 0.34 & 4.91 \\
\hline Peru & 134 & 0.45 & 0.14 & 1.41 & 134 & 0.23 & 0.03 & 1.41 \\
\hline Venezuela & 162 & 1.24 & 0.29 & 5.24 & 159 & 0.72 & 0.09 & 5.49 \\
\hline Mexico & 152 & 0.35 & 0.09 & 1.28 & 122 & 2.59 & 0.18 & 36.95 \\
\hline China & 189 & 5.22 & 0.51 & 53.94 & 202 & 0.41 & 0.12 & 1.34 \\
\hline Puerto Rico & 202 & 0.53 & 0.22 & 1.24 & 202 & 0.29 & 0.07 & 1.18 \\
\hline Pooled estimate & & $0.67 *$ & 0.41 & 0.93 & & 0.73 & 0.43 & 1.21 \\
\hline & & & & & & & & \\
\hline
\end{tabular}

Note: model II adjusted for sex, marital status, education, cognitive impairment, functional dependency, behavioral problem of older person, time spent helping with ADLs, household size, number of household asset

OR=odd ratio; $95 \mathrm{Cl}=95 \%$ confidence interval

${ }^{*} p$-value $<0.05$ 


\section{Country}

Psychological morbidity

n

OR

$95 \% \mathrm{Cl}$

$0.0 \%$

Heterogeneity $P^{2}$

Note: model II adjusted for sex, marital status, education, cognitive impairment, functional dependency, behavioral problem of older person, time spent helping with ADLs, household size, number of household asset

OR=odd ratio; $95 \mathrm{Cl}=95 \%$ confidence interval

${ }^{*} p-$ value $<0.05$

\section{Discussion}

According to demographic data not only the percentage of older people has been increasing, but also the prevalence of older caregivers, particularly in low and middle-income countries [38]. In this study, overall, the percentage of household with older caregivers (aged 65 years and over) was lesser prevalent than of the households with younger caregivers across all countries. However, the prevalence of older caregiver household in Puerto Rico and China were almost half ( $45.2 \%$ and $40.2 \%$, respectively) of the total household with older people. When compared with the high-income country such as United States of America [39], Puerto Rico and China showed a higher prevalence of older caregivers. This might reflect patterns of demographic ageing, with the rate of ageing being faster in Puerto Rico and China than in other low and middle-income countries [40], At the time of survey, median age of population of these two counties was high [41]. Likewise, increased international migration of younger age household members in these two countries might have contributed to a decrease in available young caregivers resulting in older caregivers serving in this role.

This study investigated the association of baseline caregiver age and caregiver psychological morbidity and care burden. Overall, the findings of multivariate analysis indicated that older caregiver was not associated with higher psychological morbidity and care burden across all countries. However, the pooled estimates of multivariate analysis for all countries of this study revealed that older caregivers were associated with significantly lower risk of psychological morbidity compared with younger caregivers. Thus, the findings did not support our research hypotheses that older caregivers experience worse psychological morbidity and care burden compared to younger caregivers. This is inconsistent with previous studies which indicated that higher levels of burden were associated with increased caregiver age $[42,43]$. The possible explanation for this is that caregivers may have different issues depending on their age.

Younger caregivers, as well as dealing with caregiving issues, are also often in the midst of transitions into careers and/or parenthood. Becoming a caregiver can impact a young adult's ability to form intimate relationships, engage in family life, or obtain career goals [44]. Maintaining a career while providing care 
has been associated with strain [45] and young adult caregivers reported difficulties in managing both responsibilities $[44,46]$. Combining looking after young children with caring for dependent older loved ones, sometimes called 'sandwich carer', are associated with younger populations. The insights of the analysis in this study showed that the younger caregivers had a higher number of children (aged lower than 16 years) in their households than the older caregivers [see Additional file 1]. Yet it is because of these role demands that many younger caregivers reported significantly more strain and psychological stress from lack of personal resources, such as time and energy, and career disruption $[15,47,48]$.

Apart from fewer multiple role demands and less time constraints, older caregivers perceived lower stress and burden than younger caregivers due to more personal satisfaction [49]. The developmental stage of older age usually involves a transition to retirement, and goals of work performance and achievement typically shift to finding goals that give one a sense of inner fulfillment and maintaining valued relationships. Having a closer relationship to the older person as a partnership or providing care to a spouse means a decreased burden [50]. Older caregivers, usually the spouse, compared to younger caregivers, who more often are children, had ability to see the positive rewards of caring to their beloved older household members (Prince et al., 2012). However, psychological morbidity numbers were very small for China. This undermines statistical power for estimating with precision, thus leading to overestimate the odds ratios and very wide confidence intervals, which may also have affected the care burden data for Mexico. A large odds ratio with wide confidence intervals usually happens when the prevalence of event of interest (e.g., psychological morbidity, care burden) is very low in a sample [51].

To the authors knowledge this is the first multi-country study on LMICs using standardized questionnaires to investigate the effect of caregiver age on psychological morbidity and care burden across a range of cultures. However, there are limitations; firstly, the data of this study is a cross-sectional design which is unable to predict a true value of the investigated factors. Prior mental health status of the caregivers or how this may change over time is unable to be determine. Secondly, the study is limited to households where a main caregiver provided care for one older person in the household. Thirdly, there were no available data on physical health status and social support of the caregivers, which should be consider as the mediators for adjustment of care burden. Lastly, the study used a quantitative approach to enable comparison of findings across countries. In order to have a more complete analysis, future studies should consider a qualitative approach. In-depth interviews may help to obtain greater details on the quality of the relationships within household and attitudes towards care that might affect caregivers' mental health. Further work is required to explore the psychological morbidity among younger caregivers in providing care to older people, and the identification of factors affecting the psychological morbidity in order to obtain suggestions for improving the mental health of caregivers of older people in LMICs.

\section{Conclusion}

The distribution of the household with older caregivers was inconsistent across the seven LMICs. However, the prevalence of the household with older caregivers is almost the same level as of the households with younger caregivers in Puerto Rico and China. The unadjusted and adjusted models for 
all potential covariates showed that there was no statistically significant difference in mental health outcomes in terms of psychological morbidity and care burden between younger and older caregivers across countries. The adjusted pooled estimates, however, indicated lower psychological morbidity among older caregivers. This information may help to provide a greater understanding for service support, policy makers and health care providers to plan efficient support programmes considering all caregiver age groups.

\section{Abbreviations}

LMICs: Low and Middle-Income Countries; DRG: Dementia Research Group; SRQ: Self-Reporting Questionnaire; WHO: World Health Organization; ZBI: Zarit Burden Inventory; CERAD: Consortium to Establish a Registry for Alzheimer's Disease; CSI-D: Community Screening Instrument for Dementia; GMS: Geriatric Mental State examination; DSM-IV: Diagnostic and Statistical Manual of Mental DisordersFourth Edition; ADL: Activities of Daily Living; WHO-DAS: World Health Organization - Disability assessment schedule; NPI-Q: Neuropsychiatric Inventory-Questionnaire; OR: Odd Ratio; Cl: Confidence Interval

\section{Declarations}

\section{Ethics approval and consent to participate}

Ethical approval was provided by King's College London Research Ethics Committee and by local ethical review boards in each country. All the participants read and signed the informed consent forms.

\section{Consent for publication}

Not applicable.

\section{Availability of data and materials}

Please contact the 10/66 Dementia Research Group, King's College London, United Kingdom for data requests.

\section{Competing interests}

The authors have declared that no competing interest exist.

\section{Funding}


This work was supported by the Thailand Research Fund through the Royal Golden Jubilee Ph.D. Programme [PHD/0167/2557 to RP and PV]; and the Newton Fund Ph.D. Placement for Scholars 2017/2018 [GA/PHD/Scholar/Year4/011 to RP].

\section{Authors' contributions}

RP prepared the first draft, investigated, formal analysis, and was major contributor in writing the manuscript. RM conceptualized, supervised on analyzing and interpreting results, reviewed and editing the manuscript. RV and MP conceptualized, reviewed the manuscript, and provided further contributions. KCC supervised on analyzing and interpreting results, review and editing the manuscript. All authors read and approved the final manuscript.

\section{Acknowledgments}

This is a secondary analysis of data collected by the 10/66 Dementia Research Group (www.alz.co.uk/1066/). The 10/66 DRG is led by Martin Prince from Institute of Psychiatry, Psychology and Neuroscience, King's College London. The other principal investigators, data custodians and responsible for research governance in each site are Juan Llibre Rodriguez (Cuba), Daisy Acosta (Dominican Republic), Mariella Guerra (Peru), Aquiles Salas (Venezuela), Ana Luisa Sosa (Mexico), Ivonne Jimenez (Puerto Rico) and Yueqin Huang (China). We would like to thank Jim Richards at University Central Lancashire for helpful feedback on a draft manuscript.

\section{References}

1. World Health Organization. Facing the Facts: Chronic Diseases in Low and Middle Income Countries. Geneva: World Health Organization; 2005.

2. Bluestone K, Escribano J, Horstead K. Facing the facts: The truth about ageing and development. Age International: London. 2015. https://www.ageinternational.org.uk/globalassets/documents/ageinternational-facing-the-facts-report.pdf. Accessed 15 Sep 2018.

3. United Nations, Department of Economic and Social Affairs, Population Division. World Population Prospects 2019: Highlights. New York: United Nations. 2019. https://population.un.org/wpp/Publications/Files/WPP2019_Highlights.pdf. Accessed 12 Jan 2019.

4. Schneider J, Murray J, Banerjee S, Mann A. EUROCARE: a cross-national study of co-resident spouse carers for people with Alzheimer's disease: I-factors associated with carer burden. Int J Geriatr Psychiatry. 1999 Aug;14(8):651-61. doi: 10.1002/(SICl)1099-1166(199908)14:8<651::AIDGPS992>3.0.CO;2-B

5. Li M, Dai H. Determining the primary caregiver for disabled older adults in Mainland China: spouse priority and living arrangements. J Fam Ther. 2019;41(1):126-41. doi:10.1111/1467-6427.12213. 
6. Pinquart M, Sörensen S. Spouses, adult children, and children-in-law as caregivers of older adults: a meta-analytic comparison. Psychol Aging. 2011;26(1):1.

7. $10.5175 / J S W E .2008 .773247702$

Schulz R, Sherwood PR. Physical and mental health effects of family caregiving. J Soc Work Educ. 2008 Sep 1;44:105 - 13. doi: 10.5175/JSWE.2008.773247702.

8. Zarit SH, Reever KE, Bach-Peterson J. Relatives of the impaired elderly: correlates of feelings of burden. Gerontologist. 1980;20(6):649-55. doi.org/10.1093/geront/20.6.649.

9. Garlo K, O'Leary JR, Van Ness PH, Fried TR. Burden in Caregivers of Older Adults with Advanced Illness. J Am Geriatr Soc. 2010;58(12):2315-22. doi:10.1111/j.1532-5415.2010.03177.x.

10. Christensen K, Doblhammer G, Rau R, Vaupel JW. Ageing populations: the challenges ahead. Lancet. 2009;374(9696):1196-208. doi.org/10.1016/S0140-6736(09)61460-4.

11. Rinaldi P, Spazzafumo L, Mastriforti R, Mattioli P, Marvardi M, Polidori M, et al. Predictors of high level of burden and distress in caregivers of demented patients: results of an Italian multicenter study. Int J Geriatr Psychiatry: A journal of the psychiatry of late life allied sciences. 2005;20(2):16874.

12. Serrano-Aguilar P, Lopez-Bastida J, Yanes-Lopez V. Impact on health-related quality of life and perceived burden of informal caregivers of individuals with Alzheimer's disease. Neuroepidemiology. 2006;27(3):136-42.

13. Schulz R, O'Brien AT, Bookwala J, Fleissner K. Psychiatric and physical morbidity effects of dementia caregiving: prevalence, correlates, and causes. Gerontologist. 1995;35(6):771-91. doi.org/10.1093/geront/35.6.771.

14. Donaldson C, Tarrier N, Burns A. The impact of the symptoms of dementia on caregivers. $\mathrm{Br} \mathrm{J}$ Psychiatry: the journal of mental science. 1997;170:62-8. doi:10.1192/bjp.170.1.62.

15. Carter JH, Lyons KS, Stewart BJ, Archbold PG, Scobee R. Does age make a difference in caregiver strain? Comparison of young versus older caregivers in early-stage Parkinson's disease. Mov Disord. 2010;25(6):724-30. doi.org/10.1002/mds.22888.

16. Fauth E, Hess K, Piercy K, Norton M, Corcoran C, Rabins P, et al. Caregivers' relationship closeness with the person with dementia predicts both positive and negative outcomes for caregivers' physical health and psychological well-being. Aging Ment Health. 2012;16(6):699-711. doi:10.1080/13607863.2012.678482.

17. Torti FMJ, Gwyther LP, Reed SD, Friedman JY, Schulman KA. A Multinational Review of Recent Trends and Reports in Dementia Caregiver Burden. Alzheimer Dis Assoc Disord. 2004;18(2):99-109. doi:10.1097/01.wad.0000126902.37908.b2.

18. Prince $M$, The 10/66 Dementia Research Group. Care arrangements for people with dementia in developing countries. Int J Geriatr Psychiatry. 2004;19(2):170-7. doi:10.1002/gps.1046.

19. Brinda EM, Rajkumar AP, Enemark U, Attermann J, Jacob KS. Cost and burden of informal caregiving of dependent older people in a rural Indian community. BMC Health Serv Res. 2014;14(1):207. doi:10.1186/1472-6963-14-207. 
20. Prince $M$, Brodaty H, Uwakwe R, Acosta D, Ferri CP, Guerra M, Huang Y, Jacob KS, Llibre Rodriguez JJ, Salas A, Sosa AL. Strain and its correlates among carers of people with dementia in low-income and middle-income countries. A 10/66 Dementia Research Group population-based survey. Int J Geriatr Psychiatry. 2012;27(7):670-82. doi.org/10.1002/gps.2727.

21. Prince M, Ferri CP, Acosta D, Albanese E, Arizaga R, Dewey M, et al. The protocols for the $10 / 66$ dementia research group population-based research programme. BMC Public Health. 2007;7(1):165. doi:10.1186/1471-2458-7-165.

22. Prina AM, Acosta D, Acosta I, Guerra M, Huang Y, Jotheeswaran AT, Jimenez-Velazquez IZ, Liu Z, Llibre Rodriguez JJ, Salas A, Sosa AL. Cohort profile: the 10/66 study. Int J Epidemiol. 2017;46(2):406-i. doi:10.1093/ije/dyw056.

23. Mari JD, Williams P. A comparison of the validity of two psychiatric screening questionnaires (GHQ12 and SRQ-20) in Brazil, using Relative Operating Characteristic (ROC) analysis. Psychol Med. 1985;15(3):651-9. doi:10.1017/S0033291700031500.

24. Beusenberg $\mathrm{M}$, Orley $\mathrm{JH}$, World Health Organization. A User's guide to the self reporting questionnaire (SRQ). Geneva: World Health Organization; 1994.

25. Zarit S, Orr NK, Zarit JM. The hidden victims of Alzheimer's disease: Families under stress: NYU Press, 1985.

26. Hébert R, Bravo G, Préville M. Reliability, validity and reference values of the Zarit Burden Interview for assessing informal caregivers of community-dwelling older persons with dementia. Canadian Journal on Aging/La Revue canadienne du vieillissement. 2000;19(4):494-507. doi:10.1017/S0714980800012484.

27. Pearlin LI, Mullan JT, Semple SJ, Skaff MM. Caregiving and the stress process: An overview of concepts and their measures. Gerontologist. 1990;1(5):5 83-94. doi:10.1093/geront/30.5.583. 30 ) .

28. Yates ME, Tennstedt S, Chang BH. Contributors to and mediators of psychological well-being for informal caregivers. J Gerontol B Psychol Sci Soc Sci. 1999;1(1):12-22. doi.org/10.1093/geronb/54B.1.P12. 54 ) .

29. Mayston R, Guerra M, Huang Y, Sosa AL, Uwakwe R, Acosta I, Ezeah P, Gallardo S, de Oca VM, Wang $\mathrm{H}$, Guerchet M. Exploring the economic and social effects of care dependence in later life: protocol for the 10/66 research group INDEP study. Springerplus. 2014;3(1):379. doi:10.1186/2193-1801-3379.

30. Filmer D, Pritchett LH. Estimating wealth effects without expenditure data-or tears: an application to educational enrollments in states of India. Demography. 2001;38(1):115-32.

31. Clark DO, Stump TE, Tu W, Miller DK. Improving the validity of activity of daily living dependency risk assessment. J Appl Gerontol. 2015;34(3):329-42. doi.org/10.1177/0733464812471894.

32. Etters L, Goodall D, Harrison BE. Caregiver burden among dementia patient caregivers: a review of the literature. J Am Acad Nurse Pract. 2008;20(8):423-8. doi:10.1111/j.1745-7599.2008.00342.x.

33. Carlson DL. Deviations from desired age at marriage: Mental health differences across marital status. J Marriage Fam. 2012;74(4):743-58. doi:10.1111/j.1741-3737.2012.00995.x. 
34. Horn EE, Xu Y, Beam CR, Turkheimer E, Emery RE. Accounting for the physical and mental health benefits of entry into marriage: A genetically informed study of selection and causation. J Fam Psychol. 2013;27(1):30. doi:10.1037/a0029803.

35. Montgomery RJ, Gonyea JG, Hooyman NR. Caregiving and the experience of subjective and objective burden. Fam Relat. 1985:19-26. doi:10.2307/583753.

36. Conlin M, Caranasos G, Davidson R. (1992). Reduction of caregiver stress by respite care: a pilot study. South Med J. 1992;85(11):1096 - 100.

37. McFall S, Miller BH. Caregiver burden and nursing home admission of frail elderly persons. $J$ Gerontol. 1992;47(2):73-9. doi:10.1093/geronj/47.2.S73.

38. World Health Organization. Home-based long-term care: report of a WHO study group. Geneva: World Health Organization. 2000. http://www.who.int/iris/handle/10665/42343. Accessed 14 Feb 2019.

39. National Alliance for Caregiving. and AARP. Family caregiving in the U.S.: Findings from a national survey. Bethesda, MD: Authors [updated Online 2016] https://www.caregiver.org/caregiver-statisticsdemographics. Accessed 7 Feb 2019.

40. United Nations. Department of Economic and Social Affairs, Population Division. World Population Prospects: The 2017 Revision. New York: United Nations; 2017.

41. United Nations, Department of Economic and Social Affairs. Population Division. World Population Prospects 2019, Online Edition. https://population.un.org/wpp/Download/Standard/Population/. Accessed 20 Mar 2020.

42. Rinaldi P, Spazzafumo L, Mastriforti R, Mattioli P, Marvardi M, Polidori MC, Cherubini A, Abate G, Bartorelli L, Bonaiuto S, Capurso A. Predictors of high level of burden and distress in caregivers of demented patients: results of an Italian multicenter study. Int J Geriatr Psychiatry: A journal of the psychiatry of late life allied sciences. 2005;20(2):168-74. doi:10.1002/gps.1267.

43. Serrano-Aguilar PG, Lopez-Bastida J, Yanes-Lopez V. Impact on health-related quality of life and perceived burden of informal caregivers of individuals with Alzheimer's disease. Neuroepidemiology. 2006;27(3):136-42. doi:10.1159/000095760.

44. Dellmann-Jenkins $M$, Blankemeyer $M$, Pinkard $O$. Incorporating the elder caregiving role into the developmental tasks of young adulthood. Int J of Aging Hum Dev. 2001;52(1):1-18. doi.org/10.2190/FGQA-65FU-JGNT-6C9J.

45. Zarit SH, Zarit JM. Family caregiving. In Psychology and Geriatrics, Academic Press; 2015. p. 21-43.

46. Dellmann-Jenkins $M$, Brittain L. Young adults' attitudes toward filial responsibility and actual assistance to elderly family members. J Appl Gerontol. 2003;22(2):214-29. doi.org/10.1177/0733464803022002003.

47. Harden J. Developmental life stage and couples' experiences with prostate cancer: a review of the literature. Cancer Nurs. 2005;28(2):85-98.

48. Nijboer $C$, Triemstra M, Tempelaar R, Mulder M, Sanderman R, van den Bos GA. Patterns of caregiver experiences among partners of cancer patients. Gerontologist. 2000;40(6):738-46. doi.org/10.1093/geront/40.6.738. 
49. Kim Y, Baker F, Spillers RL. Cancer caregivers' quality of life: effects of gender, relationship, and appraisal. J Pain Symptom Manage. 2007;34(3):294-304. doi:10.1016/j.jpainsymman.2006.11.012.

50. Andrén S, Elmståhl S. Relationships between income, subjective health and caregiver burden in caregivers of people with dementia in group living care: a cross-sectional community-based study. Int J Nurs Stud. 2007;44(3):435-46. doi.org/10.1016/j.ijnurstu.2006.08.016.

51. Nemes S, Jonasson JM, Genell A, Steineck G. Bias in odds ratios by logistic regression modelling and sample size. BMC Med Res Methodol. 2009;9(1):56. doi:10.1186/1471-2288-9-56.

\section{Supplementary Files}

This is a list of supplementary files associated with this preprint. Click to download.

- Additionalfile1.pdf 